\title{
La marginación social y la perspectiva de la educación privada en México. Un estudio cualitativo
}

\author{
[Social marginalization and views of private education in Mexico. A \\ qualitative study]
}

\author{
Oscar A. Martínez-Martínez (1) \& M. Elena Vega Torres (D) \\ Universidad Iberoamericana, Ciudad de México
}

\begin{abstract}
Resumen
El artículo responde a tres preguntas ¿qué tipo escuelas se considera mejor, públicas o privadas de acuerdo al nivel de marginación? ¿cuáles son los motivos para señalar que una es mejor que otra? ¿Estudiar en una escuela pública o privada puede afectar el aprendizaje de los alumnos, según la perspectiva de los entrevistados? Para responder a ellas se usaron entrevistas en profundidad que fueron analizadas según los niveles de marginación de los entrevistados, utilizando la teoría fundamentada. Los resultados muestran que, independiente del nivel de marginación, en el imaginario colectivo se ha permeado que las escuelas privadas son mejores que las públicas, los entrevistados lo atribuyen principalmente a la infraestructura, preparación de los profesores y la enseñanza del inglés; si bien estos aspectos resultaron importantes, también se consideró que el esfuerzo del alumno en sus estudios es el factor prioritario en el aprendizaje.
\end{abstract}

Palabras clave: marginación, metodología cualitativa, tipos de escuelas, aprendizaje, México.

\begin{abstract}
The present article addresses three questions. According to levels of marginalization, which type of school is considered better - public or private? On what basis can one type be said to be better than the other? According to the interviewees, does attendance of a public or private school affect students' learning? To answer these questions, in-depth interviews were conducted, and the resulting data analyzed in terms of the marginalization levels of the interviewees, using the grounded theory. The results show that regardless of the level of marginalization, the idea that private schools are better than public ones has come to dominate the collective imagination. The interviewees attributed the superiority of private education to improved infrastructure, better teacher preparation, and the teaching of English. However, while each of these aspects was considered important, the individual student's effort in their studies was considered the primary factor in learning.
\end{abstract}

Keywords: marginalization, qualitative methodology, types of schools, learning, Mexico.

Contacto: La comunicación sobre este artículo debe ser enviada a Oscar A. Martínez-Martínez, email: oscar.martinez@ibero.mx

Financiamiento: Este trabajo fue financiado por el Instituto de Investigación para el Desarrollo con Equidad (EQUIDE) de la Universidad Iberoamericana Fondo No. 0060 EQUIDE. 


\section{INTRODUCCIÓN}

La educación se define como un proceso social que transmite cultura, valores, conocimientos, prácticas, creencias, tecnologías, usos o costumbres que para una sociedad se consideran valiosos (Secretaría de Educación Pública [SEP], 2017). A través de ella es posible el aprendizaje significativo en la vida presente y futura, y se considera como una las principales vías para la movilidad social, al permitir el progreso individual y colectivo (Schmelkes, 2014).

En México, la educación es una prerrogativa constitucional, la cual debe otorgarse gratuitamente y en condiciones de igualdad -las personas gozan de sus derechos independientemente de sus diferencias por sexo, económicas o culturales (SEP, 2017). El Artículo 3 de la constitución establece que el Estado debe ofertar servicios educativos en los niveles preescolar (dos años), primaria (seis años), secundaria (tres años), media superior (tres años) y superior (cuatro o cinco años), a excepción de la última, todas tienen carácter de obligatorio. Para atender este mandato, el sistema educativo se implementa en zonas urbanas, rurales o indígenas, en donde existen distintos tipos de escuelas, por ejemplo, en preescolar y primaria, además de urbanas, hay escuelas indígenas y comunitarias; en secundaria, las técnicas y telesecundarias; en media superior bachilleratos y formación técnica (Instituto Nacional para la Evaluación de la Educación [INEE], 2004).

En las últimas dos décadas México ha tenido avances en el incremento de la matrícula en las escuelas, sobre todo en los servicios educativos obligatorios, especialmente, entre quienes pertenecen a niveles socioeconómicos bajos, pues las tasas de escolarización han aumentado de manera importante y, en el caso de los grupos de nivel socioeconómico medio y alto, son casi universales (Schwartzman, 2005). Lo anterior se atribuye a la redirección de las políticas económicas y sociales (López, Alessandre, \& Pla, 2006; Torres \& Tenti, 2000). Debido a que en México existe una diversidad de escuelas, los servicios educativos se caracterizan por ser heterogéneos en el desempeño de sus estudiantes, profesorado, infraestructura, organización escolar y el currículum que diseñan y ejecutan (Murillo, 2003). La oferta y la calidad de los currículums dependen de las zonas geográficas en donde se ubiquen (Ministerio de Educación, 2006).

Como parte de la expansión de la oferta educativa, la matrícula en las escuelas privadas se ha incrementado también, concentrándose principalmente en zonas urbanas y algunas semiurbanas, es decir, donde existen bajos niveles de marginación territorial. Los servicios que ofrecen son heterogéneos, pues dependen de aspectos como la ubicación o el segmento de población que quieren atraer. En ese sentido, que una escuela sea privada no implica automáticamente que ofrezca los métodos de enseñanza más innovadores o la mejor infraestructura.

Por lo anterior, el presente artículo aporta elementos de la construcción en el imaginario colectivo sobre las escuelas privadas, 
contestando las siguientes incógnitas ¿qué tipo escuelas se considera mejor de acuerdo al nivel de marginación? ¿cuáles son los motivos para señalar que una es mejor que otra? ¿Estudiar en una escuela pública o privada puede afectar el aprendizaje de los alumnos según la perspectiva de los entrevistados?

Panorama de la educación pública y privada en México.

Las escuelas públicas y privadas varían en objetivos, métodos de enseñanza, orientación laica o no laica, ubicación geográfica, por señalar algunas. La primera de ellas es financiada con recursos públicos, recaudados de los impuestos de los ciudadanos, administrada y supervisada por instituciones públicas. Las privadas pueden recibir o no subvenciones del gobierno, son administradas por la iniciativa privada y pueden regirse bajo sus normas, para ingresar y permanecer en ellas es necesario el pago de cuotas o colegiaturas (Suárez, 2012).

En México $86.4 \%$ de las escuelas son parte del sector público y $13.6 \%$ son privadas (INEE, 2015). En educación básica 90\% de estudiantes se encuentran en escuelas públicas y el resto en privadas. En educación superior, la oferta educativa ha tenido grandes desafíos pues cubre entre el 33 y 34\% (Guzmán, 2012). Por su parte, la matrícula de licenciatura o pregrado que es atendida por universidades privadas creció en $164.5 \%$, en la década de los 90's pasó de 198,207 estudiantes a 525,058 al finalizar esa década (Muñoz \& Silva, 2013). Esto significó una tasa de crecimiento anual promedio de $15 \%$, misma que quintuplicó el ritmo experimentado por el sector público. Al finalizar ese periodo, este tipo de instituciones atendían el 30\% de la matrícula de licenciatura. Lo mismo sucedió con la oferta de posgrados, la matricula se incrementó casi cinco veces en las escuelas privadas (Muñoz \& Silva, 2013).

La distribución de las escuelas públicas y privadas también obedece a la zona geográfica. Por una parte, las públicas grandes cuentan con mayores recursos, mejores materiales y se ubican en áreas urbanas, mientras que las ubicadas en zonas rurales o con altos índices de marginación tienen recursos escasos (INEE, 2004). Por otra, las escuelas privadas tienen más presencia en las zonas urbanas (Fernández, 2008) y ofrecen mejor infraestructura escolar, un mejor equipamiento didáctico, una planta docente con mejor perfil profesional, una matrícula de estudiantes con niveles socioeconómicos medios o altos, una menor incidencia de inasistencias docentes, un mayor número de clases efectivas al año, un horario más extenso de clases, un currículo enriquecido, y padres de familia más demandantes y participativos, entre otras cosas (INEE, 2007), aspectos que pueden influir en las decisiones de los padres para inscribir a sus hijos en ellas.

En cuanto al logro educativo las pruebas estandarizadas aplicadas en el país como son los exámenes nacionales del logro académico en centros escolares (ENLACE), exámenes de la calidad y el logro educativo (EXCALE) y plan nacional para la evaluación de los aprendizajes (PLANEA), muestran que los estudiantes de escuelas privadas tienen mejor dominio en 
español y matemáticas, en lo que respecta a educación básica y media básica (INEE, 2015). A pesar de ello, es importante considerar las diferencias entre las zonas geográficas y a las condiciones demográficas, sociales y económicas que generan servicios educativos heterogéneos (INEE, 2004). En ese mismo sentido, los resultados del programa para la evaluación internacional de alumnos (PISA) muestran que el desempeño académico de los estudiantes en una escuela pública o privada depende más de la condición socioeconómica de sus familias (Organización para la Cooperación y Desarrollo Económico [OCDE], 2011).

El aumento de la oferta educativa privada en México como en distintos países de América Latina, se asocia a tres motivos: 1) las limitaciones de las escuelas públicas para incorporar estudiantes; 2) se responde a demandas diferenciadas de la población, por ejemplo, interés en por la educación religiosa, priorizar en los idiomas, entre otras cosas; 3) el crecimiento de la clase media, cuyos recursos económicos se elevaron y ha tenido interés por distanciarse de la oferta educativa gubernamental, o bien por la idea de que la educación privada es de mayor calidad (Murillo \& Martinez, 2017).

Distintos estudios dan cuenta de cómo en el imaginario social está instalada la idea que las escuelas privadas ofrecen una educación de mayor calidad que las públicas (Murillo \& Martinez, 2017). Canales, Bellei, y Orellana (2016) muestran que las familias deciden por este tipo de escuelas, esencialmente, por razones sociales, pues buscan separarse de grupos socioeconómicamente inferiores -considerados riesgosos-, lo cual les hace descartar escuelas públicas, gratuitas y no selectivas, y valorar escuelas que ofrecen un fuerte control disciplinario (Fernández, 2008).

Para los padres las escuelas privadas ofrecen servicios distintos a las públicas, pues consideran que las primeras atraen a alumnos de alto rendimiento y mejores profesores (OCDE, 2011). Entre los países que pertenecen a la Organización para la Cooperación y Desarrollo Económico (OCDE) los padres eligen ese tipo de escuelas ante la probabilidad de que sus hijos tengan como compañeros a estudiantes que tienen una posición económica similar o más elevada, que los recursos profesores y materiales serán de mejor calidad y que hay más orden. Con estas ideas se tiene la disposición de pagar por los servicios educativos, aun cuando puedan existir escuelas públicas que posean las mismas características, incluso estudiantes que tengan ventajas económicas (Canales et al., 2016; Vinacur, 2016).

De igual forma, una razón para optar por las escuelas privadas es por su funcionamiento pues las personas tienden a relacionar que si existe buena infraestructura, también habrá calidad educativa (Sarramona, 2004). Lo cual pone en desventaja a las escuelas públicas, al menos en el caso mexicano, pues estas tienen diversos desafíos en esta materia, ya que las medidas compensatorias instrumentadas para disminuir esta desigualdad han carecido de éxito (Guevara Niebla, Muñoz Izquierdo, Arizmendi, \& Romo, 2012) especialmente en las áreas rurales y semiurbanas. 


\section{MÉTODO}

La investigación se realizó en cuatro estados que representan un nivel distinto de bienestar social de acuerdo a la medición de Martínez-Martínez, Lombe, Vazquez-Rodriguez, y Coronado-Garcia (2016); a saber: Ciudad de México (muy alto), Tamaulipas (alto), Estado de México (medio) y Oaxaca (bajo).

$\mathrm{Al}$ interior de cada estado se seleccionaron municipios tomando en cuenta variables como: tipo de municipio (rural o urbano), índice de marginación y cantidad poblacional. La finalidad era tener una serie de contextos heterogéneos entre sí, pues de acuerdo con algunos estudios cualitativos (Martínez, 2017; Rodríguez, Flores, \& Jiménez 2003) la diversidad de aspectos socioeconómicos, culturales y geoespaciales que distinguen los lugares y perfiles de entrevistados incluidos, favorece la amplitud y la posibilidad de aprender, y permite que al saturarse las categorías se encuentren los hilos conductores que las unen dentro de la heterogeneidad.

En total se realizaron 245 entrevistas en profundidad en 71 municipios (16 en Ciudad de México, 6 en Tamaulipas, 31 en Estado de México y 18 en Oaxaca). De ellas, 76 fueron en la Ciudad de México, 44 en Tamaulipas, 53 en el Estado de México y 72 en Oaxaca. En la selección de los entrevistados ocupamos la técnica de bola de nieve. Con el propósito de privilegiar la heterogeneidad antes señalada, las entrevistas se realizaron tomando en consideración aspectos como los niveles socioeconómicos, niveles educativos, edades y género. Respecto a este último indicador se buscó proporción entre hombres y mujeres, como se puede ver en la Tabla 1.

Tabla 1 Características de los entrevistados

\begin{tabular}{llcc}
\hline Estado & & $\%$ & Edad Media \\
\hline Ciudad de México & Hombre & 46.2 & 42.53 \\
& Mujer & 53.8 & 43.52 \\
Tamaulipas & Hombre & 50 & 47.86 \\
& Mujer & 50 & 44.09 \\
Estado de México & Hombre & 50.9 & 48.07 \\
& Mujer & 49.1 & 43.77 \\
Oaxaca & Hombre & 50 & 44.14 \\
& Mujer & 50 & 45.69 \\
\hline
\end{tabular}

La guía de entrevista y el protocolo de investigación fueron aprobados por el comité de ética de la Universidad Iberoamericana. Las entrevistas fueron grabadas con permiso de los entrevistados por medio de un consentimiento informado, y transcritas, posteriormente capturadas en el programa Nvivo para analizar las categorías y subcategorías, lo que permitió identificar tendencias y determinar el momento en que ya estaban saturadas.

El método de análisis empleado fue la teoría fundamentada (Glaser \& Strauss 1967), la cual tiene como característica la fundamentación de conceptos a partir de los datos empíricos (Strauss $\&$ Corbin, 1998), de tal forma que se establece el trabajo de campo como la fuente principal del conocimiento. De este modo, nuestra investigación partió inductivamente de los datos empíricos recogidos a partir de las entrevistas en profundidad para reinterpretar las 
aproximaciones teóricas existentes y las particularidades del contexto mexicano.

Índice de marginación

La investigación utilizó como medio de comparación el índice de marginación municipal elaborado por el Consejo Nacional de Población de México (Consejo Nacional de Población [CONAPO], 2015), que se construye a partir de cuatro dimensiones: (a) educación (analfabetismo, población que no ha terminado la escuela primaria); (b) viviendas (casas sin servicios de plomería o sanitarios, hogares sin electricidad, hogares sin tuberías de agua, hogares con cualquier nivel de hacinamiento, casas con piso de tierra); (c) distribución de la población (ciudades con menos de 5,000 habitantes); y (d) ingreso monetario (población laboral que gana hasta dos veces el salario mínimo).

Con las variables anteriores y utilizando la técnica de estratificación óptima, se crea el índice con cinco clasificaciones territoriales: muy alto, alto, medio, bajo y muy bajo, (CONAPO, 2016). Un contexto de alta marginación es el más vulnerable porque generalmente presenta porcentajes más altos de carencias de los indicadores con los que se conforma. Por el contrario, los lugares con una marginación muy baja tienen porcentajes muy bajos de indicadores de exclusión.

\section{RESULTADOS}

En este apartado se muestran los resultados del análisis de las entrevistas a profundidad, que permitieron responder a las preguntas ¿de acuerdo al nivel de marginación, qué tipo escuelas se considera mejor? ¿cuáles son los motivos para señalar que una es mejor que otra? ¿Estudiar en una escuela pública o privada puede afectar el aprendizaje de los alumnos, según la perspectiva de los entrevistados?

Percepción sobre las escuelas

Los hallazgos muestran que, independiente del nivel de marginación, existe una clara preferencia por las escuelas privadas. Por una parte, los entrevistados en contextos de baja y muy baja marginación, que son aquellos que tienen mayor poder adquisitivo, prefieren este tipo de escuelas porque estudiaron ahí o porque sus hijos ahora estudian en esas escuelas, sobre todo esto último, cuando los padres ahora tienen mayores recursos que la generación anterior (abuelos), pudiendo pagar por la educación de sus hijos:

Pues siento que la educación privada a nivel básico está mejor, ¿no?, porque tienen inglés, tienen computación, tienen también otros idiomas a veces, francés o alemán. Tienen otras actividades y además tienen mayor vigilancia (Entrevista, Persona de muy baja marginación).

Siempre he estado en escuelas públicas, sin embargo, desde mi experiencia, las escuelas públicas no enseñan a los niños de niveles 
básicos algunas habilidades que después es más difícil adquirir, como el dominio de idiomas o de la tecnología (Entrevista, Persona de muy baja marginación).

En las escuelas públicas [...] las materias solamente tienes una vez para aprovecharlas y las escuelas privadas hay un poquito más de opción de repetir [...] es más personalizada la educación (Entrevista, Persona de muy baja marginación).

Por otra parte, las personas que no estudiaron en escuelas privadas, ni sus hijos estudian o han estudiado en ellas, construyen la idea que la escuela privada es mejor a partir de los comentarios de algún familiar o conocido que sí lo hizo. Un aspecto a destacar dentro de este grupo es que, en áreas rurales y semiurbanas, que son contextos de alta y muy alta marginación, a pesar de no contar con escuelas privadas en la zona (pues estas se concentran principalmente en la parte urbana) en su imaginario colectivo consideran que estas son mejores que las públicas. Así, al ser preguntados sobre qué tipo de escuela es mejor, se obtuvieron por ejemplo, las siguientes respuestas:

La privada. Pues, pienso que ha de ser mejor, ¿no? Ha de ser mejor (Entrevista, Persona de muy alta marginación).

Pues yo me imagino que la privada, ¿no? Un poquito más de educación (Entrevista, Persona de alta marginación).

A pesar de que se considera a la privada como mejor, también se reconoce independientemente del nivel de marginación, que la educación es un derecho, por tanto, el gobierno tiene obligación de proporcionar escuelas públicas y gratuitas.
Motivos para preferir un tipo de escuela

Las evidencias muestran que los entrevistados consideran que las escuelas privadas son mejores por las condiciones de la infraestructura y currículum, así como cursos o clases adicionales como las de idiomas o computación, los cuales se imparten desde la educación básica, entre otros aspectos, como se leen en las siguientes evidencias.

\section{a) Exigencia a la escuela}

El principal motivo es poder exigir a las autoridades de la escuela y a los maestros debido a que se paga por el servicio. Es decir, los entrevistados consideran que los servicios obtenidos son mejores o de mayor calidad debido a la existencia de un pago de por medio. Esta perspectiva está presente independientemente si el entrevistado o alguien de su familia estudió o no en escuela privada:

Me imagino yo que la privada es mejor, porque se está pagando por algo, para que se enseñe mejor (Entrevista, Persona de muy baja marginación).

[...]considero que la educación privada es buena no sé si sea de mejor calidad nunca he asistido a una de ellas, solo sé que con dinero todo es mejor (Entrevista, Persona de alta marginación).

La privada digo ¿no? Porque ahí hay que pagar, bueno eso lo que yo digo ehh, no sé (Entrevista, Persona de media marginación). 


\section{b) Desempeño del profesorado}

Las personas entrevistadas mencionaron que en las escuelas privadas los grupos son más reducidos permitiendo una atención más personalizada a los alumnos, esto, además, conlleva a una mayor exigencia a los alumnos. Asimismo, señalaron que los profesores no faltan a sus centros laborales, pues su tipo de contratación es diferente a los que tienen los maestros en las escuelas públicas:

Considero que en las privadas les ponen más atención a los jóvenes o a los niños que en una pública (Entrevista, Persona de baja marginación).

La educación privada, porque hay más exigencia de los maestros a los alumnos (Entrevista, Persona de alta marginación).

La privada. Porque hay mayor atención, hay más maestros, es una idea (Entrevista, Persona de baja marginación).

La privada. ¿Por qué? Ehh... hay mayor atención en los niños en la escuela privada que en la escuela pública ehh... por lo que me comentan algunos familiares o amigos (Entrevista, Persona de muy baja marginación).

Aparte que se reconoce que en las privadas no hay paros laborales por parte de los maestros, cosa que en las públicas es frecuente, sobre todo en el estado de Oaxaca, debido a las movilizaciones sindicales:

Porque es una escuela particular en donde no tienen paros, ehh tienen clases de lunes a viernes y este se refleja con la educación que ha obtenido mi hija (Entrevista, Persona de baja marginación).
Pues yo pienso que las dos son buenas, no más que por lo mismo de los paros, yo pienso que las privadas (Entrevista, Persona de baja marginación).

Aprendizaje y tipo de escuela

Los entrevistados en casi todos los niveles de marginación coinciden que el aprendizaje depende del estudiante, así como de la actitud y el empeño que ponga en sus estudios y no tanto del tipo de escuela a la cual asiste, aun cuando se reconoce que las privadas tienen mejor calidad de infraestructura, profesores y las clases adicionales como el inglés:

Porque, aunque te paguen muy buenas escuelas si tú no quieres estudiar pues no aprendes y si vas a una pública que es de gobierno, aunque sea pobremente si tienes interés realmente en estudiar y aprender, aprendes, si no pues no; eso es dependiendo de cada quien (Baja marginación).

Yo creo que depende del alumnado porque un alumno puede ir a la mejor escuela pagada y si no la hace pues no es, en cambio un muchacho de lo más sencillo o humilde que sea, yendo a una escuela de gobierno pues pone todo su empeño y sí logra destacar en algún campo (Entrevista, Persona de media marginación).

Creo que no depende de si es pública o privada sino del esfuerzo que uno ponga al estudiar (Entrevista, Persona de baja Marginación).

Por otra parte, se reconocen que el contexto influye en el aprendizaje, pues admiten que no es lo mismo estudiar en una ciudad que una zona 
rural, en este último los apoyos que reciban por parte de sus padres son limitados y pueden afectar el desempeño escolar:

La educación no puede ser igual en un pueblo, en una ciudad que en un rancho (Entrevista, Persona de baja marginación).

Algunos entrevistados también señalaron que en las escuelas privadas solo asisten personas que tienen mayores recursos económicos, por esa razón, aun cuando se pusieran en su contexto sería difícil que sus hijos pudieran acudir a ellas:

Ahí solamente van a la escuela los hijos de los que tienen dinero, porque los que no tienen van a la pública (Entrevista, Persona de baja marginación).
Un aspecto a señalar es que las personas en contextos de baja y muy baja marginación, que ellos y/o sus hijos han tenido acceso a escuelas privadas reconocen que existen distintos tipos de privadas y la calidad también varía, de acuerdo a su costo:

A pesar de que van en una escuela privada no estoy muy de acuerdo en la calidad que les dan, pero en cuanto a una escuela privada es lo que puedo pagar.... me gustaría que hubiese otro tipo de... atención y que los profesores que estén más preparados (Entrevista, Persona de muy baja marginación).

\section{CONCLUSIONES}

Las percepciones de los entrevistados en los diferentes contextos de marginación muestran que las escuelas privadas son consideradas de mejor calidad, sin embargo, las razones que dan origen a estas percepciones es distinta a otros estudios como es el caso chileno (Canales et al., 2016) donde encuentran que la preferencia por estas escuelas es porque las familias buscan distanciarse de grupos que consideran socioeconómicamente inferiores, que son los que acuden a las escuelas públicas, sumado a ello, el gobierno incentiva de alguna forma el ingreso a las privadas subvencionadas por medio de un voucher, lo cual, al final, genera una segregación y la construcción de que las privadas son mejores que las públicas.
El caso mexicano es diferente, porque casi la mitad de la población vive en condiciones de pobreza y el $9.5 \%$ en pobreza extrema (Consejo Nacional de Evaluación de la Política de Desarrollo Social [CONEVAL], 2015), lo cual excluye a todas estas personas de poder solventar una educación privada, además de no existir apoyo monetario para acceder a las mismas. Lo anterior lleva a preguntarse ¿qué motiva en este contexto a considerar mejores las escuelas privadas? No podríamos confirmarlo debido a las limitaciones del estudio, pero nuestras evidencias apuntan que la construcción de esta percepción en el imaginario colectivo es porque una escuela privada representa una aspiración, por esa razón, aun cuando únicamente el $16.32 \%$ de nuestros entrevistados 
estudiaron o sus hijos estudian en este tipo de escuelas, esta idea prevalece en casi todos los sujetos de investigación.

La educación privada como un aspecto aspiracional, podría atribuirse a dos motivos según nuestras evidencias. El primero, porque se considera que al pagar por un servicio este será mejor que aquéllos en los que no hay un pago realizado por ellos, como los ofrecidos por el gobierno por medio de la educación pública y gratuita; aunque estas escuelas se pagan por los impuestos captados, por tanto, se tiende a valorar más cuando existe un desembolso económico, aun cuando el servicio puede no cubrir del todo sus expectativas o necesidades. Por esa razón, las escuelas públicas que incluso pueden ofrecer servicios similares a algunas privadas, se les resta valor. El segundo, son los desafíos que enfrenta la educación pública en México en materia de infraestructura, sobre todo en zonas rurales y semiurbanas, que pueden estar influyendo en la percepción negativa, donde además se suman las experiencias personales o de terceros.

Estas evidencias muestran que esta idea se ha penetrado a tal grado que incluso en aquellos lugares como los rurales donde no están presentes las escuelas privadas y no se tiene un punto de comparación, también se consideran las mejores opciones de estudio, pero se reconoce que no es fácil ingresar y mantenerse en ellas debido al costo que tienen. Sin embargo, se considera que las privadas cuentan con mejor infraestructura, nivel académico y actividades de formación que son complementarias para la educación, aspectos que las escuelas públicas no tienen; además, se considera que los profesores cuentan con mejor preparación y su trabajo es más personalizado.

Por otra parte, encontramos en contextos de media y baja marginación ubicados en zonas semiurbanas, que las escuelas privadas aun cuando no se consideran de alta calidad resultan una opción viable, ante la falta de cobertura en las escuelas públicas como se ha conocido a través de otros estudios (Guzmán, 2012; Muñoz \& Silva, 2013). En lugares de alta marginación se identifica que la escuela pública es valorada por ser la única opción, sin embargo, se reconoce que no cuenta con la infraestructura necesaria, pues el tamaño y los servicios que ofrece se relacionan con el nivel de urbanización donde está ubicada (INEE, 2004), es decir, para que una escuela cuente con servicios, y que estos sean adecuados para contribuir al logro educativo, influye la zona donde se ubique (Canales, et al., 2016; Fernández, 2008).

Los hallazgos en el estudio si bien dan una perspectiva sobre el imaginario colectivo de los entrevistados, los resultados de las pruebas estandarizadas (INEE, 2015), en cierta forma los corroboran, pues a nivel básico y medio básico, las privadas obtienen los niveles más altos de aprovechamiento en español y matemáticas, sin embargo, es importante reconocer que este tipo de pruebas dejan fuera otras áreas como inglés, historia, ciencias sociales u otras habilidades.

En el logro educativo, si bien las escuelas tienen un papel importante, también influyen las características individuales de los estudiantes, seguidos de la modalidad educativa y los factores de composición de la escuela y, con menor fuerza, 
las características estructurales de los centros escolares (INEE, 2007). La familia funge un papel protagónico por los atributos que les concede a los estudiantes, ya que en ello influye la pertenencia social, la vestimenta, la lengua, materna, los comportamientos, es decir, se enlazan lo educativo y lo social (López et al., 2006) además que si los padres expresan interés en su desarrollo académico los estudiantes tienen mejores resultados académicos (OCDE, 2011), de ahí que la familia sea importante para que las personas alcancen estudios de nivel superior (Vinacur, 2016).

En los logros educativos también influye el ingreso de las familias (Guzmán, 2012), el contexto en el que se encuentran, así como sus recursos sociales y culturales (Guevara et al., 2012), por esa razón, los jóvenes que provienen de grupos sociales más desventajados tienen menos posibilidades de ingresar a la universidad (Guzmán, 2012). Estos factores explican en cierta medida los resultados de las pruebas estandarizadas en las escuelas privadas, además de contradecir en cierta forma la percepción de los entrevistados, cuando señalan que en el aprendizaje no influye el tipo de escuela sino lo que importa es actitud y el empeño.

Las escuelas públicas y privadas son diversas en objetivos, oferta educativa, organización institucional, calidad, tipo de poblaciones a las que se dirigen y reciben (Suárez, 2012). Además, que los datos oficiales muestran que en México casi el 90 por ciento de las escuelas son públicas, lo cual se traduce en que su cobertura es excesivamente mayor en comparación con las privadas, si bien estas últimas, han aumentado en número, contextos que abarcan y tipos de servicios que ofrecen (Murillo \& Martínez, 2017). Por los motivos señalados, el documento presenta un primer acercamiento para comprender por qué las escuelas privadas se han incorporado en el imaginario colectivo como la mejor opción sobre las públicas, aun cuando en las primeras (privadas) existen una diversidad y su calidad varia en relación al barrio donde se ubican, el costo de las mismas e incluso el sector al cual quieren abarcar, por tanto, pueden clasificarse en distintos niveles de calidad desde muy alta hasta las "escuelas privadas patito" que de acuerdo a los entrevistados son aquellas donde el pago es bajo y la calidad no es tan buena, pero a pesar de ello, estás son preferibles a una pública.

Un aspecto importante a destacar en nuestros resultados, es que los entrevistados consideran que el estudiar en una escuela privada a pesar de las ventajas que esto significa, no influye en el aprendizaje, pues lo importante es el empeño que realicen los alumnos en sus estudios, atribuyendo de esta forma, al aspecto individual y no al tipo escuela o contexto, por esa razón señalaban, que se puede ser de cualquier sector social y sobresalir; sin embargo, como muestran los resultados de las evaluaciones estandarizadas en México, las privadas tienen un mejor desempeño en las pruebas estandarizadas (INEE, 2015), lo cual según la OCDE (2011) se podría atribuir a las condición socioeconómica de las familias.

Por otra parte, no es de extrañarse que en las personas en lugares de muy baja y baja marginación, consideren como la mejor opción a 
las privadas, probablemente como un efecto de lo algunos autores (López et al., 2006; Torres \& Tenti, 2000) han atribuido a la modificación de las políticas económicas y sociales que se han implementado, donde los sectores de clase media comenzaron a optar por servicios de este tipo, más vinculados a la lógica del mercado, en ese mismo sentido, las clases medias que optan por las escuelas privadas como un medio para vincularse con personas de condiciones económicas favorables y que se asume que favorecerá las relaciones y las buenas redes. Sin embargo, un aspecto a destacar en nuestros hallazgos es que las privadas sean valoradas en las zonas de alta y muy alta marginación, donde no existen este tipo de escuelas, lo cual pudiera atribuirse a un aspecto aspiracional, por tanto, expresiones como "al tener dinero las cosas son más sencillas" se vinculan a la aspiración de contar con recursos.

La concepción de que al ofrecer servicios de idiomas y computación las escuelas privadas se vuelven la mejor opción, no necesariamente es real, porque la existencia de ese tipo de servicios no implica que se otorguen realmente en condiciones adecuadas, pues no existe una garantía que los programas conduzcan al desarrollo de habilidades y la adquisición de conocimientos, pues estos no se someten a evaluaciones estandarizadas que permitan analizar y comparar aún entre las mismas privadas.
Los resultados hasta presentados aquí llevan a una serie de implicaciones prácticas y retos para el Ministerio de Educación de México. Primero, fortalecer la infraestructura educativa en el país especialmente en los contextos de muy alta y alta marginación que son municipios rurales y semiurbanos, para garantizar un estándar mínimo de condiciones a los alumnos. Por otra parte, las clases de idiomas y computacionales, si bien los entrevistados la asocian con la calidad, estas son indispensables en un contexto como el actual donde ambas habilidades son necesarias, pero es importante que estas sean evaluadas de forma estandarizada.

Los hallazgos en esta investigación nos llevan a nuevas preguntas, como, por ejemplo: ¿cuánto puede influir la calidad de las escuelas públicas en el abandono escolar? ¿qué percepción se tiene de las escuelas públicas o privadas a nivel pregrado o posgrado? Finalmente, es importante reconocer unas limitaciones de la investigación, primero, todos los entrevistados tenían estudios, el que menos tuvo fue educación básica, por tanto, no se captó la percepción de las personas que carecen de educación escolar. Segundo, el estudio se realizó en cuatro estados de México, que, si bien son representativos del país por su nivel de bienestar social, no podríamos generalizar los resultados a todos los estados, sin embargo, las evidencias dan una clara idea sobre el imaginario colectivo en varias regiones del país. 


\section{REFERENCIAS}

Canales, M., Bellei, C., \& Orellana, V. (2016). ¿Por qué elegir una escuela subvencionada? Sectores medios emergentes y elección de escuela en un sistema de mercado. Estudios Pedagógicos, 42(3), 89-109. doi: 10.4067/S0718-07052016000400005

Consejo Nacional de Población. (2015). Índice de marginación municipal. México D. F., México: CONAPO.

Consejo Nacional de Población. (2016). Índice de marginación por entidad federativa $y$ municipio 2015. Ciudad de México, México: CONAPO.

Consejo Nacional de Evaluación de la Política de Desarrollo Social. (2015). Informe de pobreza en México 2014. Ciudad de México, México: CONEVAL.

Fernández, M. (2008). Escuela pública y privada en España: La segregación rampante. Profesorado. Revista de Curriculum y Formación del Profesorado, 12(2), 1-27.

Guevara Niebla, G., Muñoz Izquierdo, C., Arizmendi, R., \& Romo, A. (2012). Un diagnóstico global. En G. Guevara Niebla (Comp.), La cástrofe silenciosa (pp. 31-152). Ciudad de México, México: Fondo de Cultura Económica.

Guzmán, C. (2012). Quedar fuera: Experiencias y vivencias de los jóvenes que no logran ingresar. Cultura y representaciones sociales, 6(12), 131-164.

Glaser, B., \& Strauss, A. (1967). The discovery of grounded theory: Strategies for qualitative research. Chicago, IL: Aldine.

Instituto Nacional para la Evaluación de la ducación. (2004). La calidad de la educación básica en México 2004. Ciudad de México, México: INEE.

Instituto Nacional para la Evaluación de la Educación. (2007). Factores escolares y aprendizaje en México. El caso de la educación básica. Ciudad de México, México: INEE.

Instituto Nacional para la Evaluación de la Educación (2015). Panorama educativo de México. Indicadores del sistema educativo nacional 2014. Ciudad de México, México: INEE.

López, N., Alessandre, V., \& Pla, J. (2006). Educación y desigualdad social. Buenos Aires, Argentina: Ministerio de Educación, Ciencia y Tecnología.

Martínez, O. (2017). Diseño y operación de un programa de transferencias monetarias: La renta garantizada de ciudadanía, ayuntamiento de Valencia, España. Revista Desacatos, 53(1), 114-129.

Martínez-Martínez, O. A, Lombe, M., VazquezRodriguez, A., \& Coronado-Garcia, M. (2016). Rethinking the construction of welfare in Mexico: Going beyond the economic measures. International Journal of Social Welfare, 25(3), 259-272. doi: 10.1111/ijsw.12202

Ministerio de Educación. (2006). Educación y desigualdad social. Buenos Aires, Argentina: Ministerio de Educación, Ciencia y Tecnología.

Muñoz, C., \& Silva, M. (2013). La educación superior particular y la distribución de oportunidades educativas en México. Revista de la Educación Superior, 166(42), 81-121.

Murillo, J. (2003). El movimiento de investigación en eficacia escolar. La investigación sobre eficacia escolar en Iberoamérica. Revisión internacional del estado del arte. Bogotá, Colombia: Convenio Andrés Bello.

Murillo, J., \& Martinez, C. (2017). Segregación social en las escuelas públicas y privadas en América Latina. Centro de Estudios de 
Martínez-Martínez, O. A, \& Vega Torres, M. E. (2019). La marginación social y la perspectiva de la educación privada en México. Un estudio cualitativo, Revista de Sociología, 34(2), 20-33. doi: 10.5354/0719-529X.2019.51272

Educación y Sociedad, 38(140), 727-750. doi: 10.1590/ES0101-73302017167714

Organización para la Cooperación y Desarrollo Económico. (2011). PISA. In Focus. Recuperado de: http://www.oecd.org/pisa/pisaproducts/pisai nfocus/49184642.pdf

Rodríguez, G., Flores, J. G., \& Jiménez, E. G. (2003). Metodología de la investigación cualitativa. La Habana, Cuba: Editorial Félix Varela.

Sarramona, J. (2004). Factores e indicadores de calidad en la educación. Barcelona, España: Biblioteca Latinoamericana.

Schmelkes, S. (2014). Educación, pobreza y desigualdad. En P. Cloter (Coord.), Pobreza y desigualdad: Un enfoque multidisciplinario. Ciudad de Mexico, México: Universidad Iberoamericana.

Schwartzman, S. (2005). Accesos y retrasos en la educación el América Latina. En N. López \& A. Pereyra (Coord.), Equidad en el acceso y la permanencia en el sistema educativo. Buenos Aires, Argentina: IIPE-UNESCO.

Secretaría de Educación Pública. (2017). Modelo Educativo para la educación obligatoria. Ciudad de México, México: SEP.
Suárez, M. (2012). Educación superior pública y privada en México. Desigualdades institucionales y opiniones de los estudiantes. Ciudad de México, México: SES.

Corbin, J., \& Strauss, A. (1998). Basics of qualitative research: Techniques and procedures for developing grounded theory. Thousand Oaks, CA: Sage Publications, Inc. Torres, R. M., \& Tenti, E. (2000). Políticas educativas y equidad en México. La experiencia de la educación comunitaria, la telesecundaria $y$ los programas compensatorios. Ciudad de México, México: SEP.

Vinacur, T. (2016). ¿Los alumnos de las escuelas privadas están mejor preparados para ingresar a la universidad? Revista Colombiana de Educación, 70(1), 175-200. doi: 10.17227/01203916.70rce175.200

Manuscrito recibido: 06-10-2018 Manuscrito aceptado: 25-06-2019 